\title{
A new neuro-fuzzy-based classification approach for hyperspectral remote sensing images
}

\author{
Nafiseh Kakhani*(0) and Mehdi Mokhtarzade \\ K.N. Toosi University of Technology, Vali-Asr Street, Mirdamad Cross, Tehran 19967-15433, Iran. \\ *Corresponding author.e-mail: nkakhani@mail.kntu.ac.ir
}

MS received 16 January 2017; revised 18 April 2018; accepted 4 May 2018;

published online 22 January 2019

Hyperspectral images are widely used in many applications. However, finding the appropriate hyperspectral image classification technique is a challenge. In this paper, we propose a new method by using an artificial intelligence-based method for hyperspectral image classification. The system has two parts: first, a pre-processing step, which helps the training phase to work faster; and second, the training part, which consists of calculating the neuro-fuzzy parameters. The prepared system is then applied to the classification of images. Three well-known hyperspectral datasets, including Pavia University from reflective optics system imaging spectrometer, the Botswana image from Hyperion and the Indian Pine image from airborne visible/infrared imaging spectrometer, were chosen to test the method. The final results of the experiments show that this system outperforms two classical methods of hyperspectral classification: support vector machine and spectral angle mapper. The comparison of the final results was made using two different metrics: overall accuracy and total disagreement. The proposed method increases the overall accuracy by about $5 \%$ for the Pavia University dataset, $2 \%$ for the Botswana dataset and $7 \%$ for the Indian Pine dataset. The total disagreement was reduced by about 0.01 for the Pavia University, 0.03 for the Botswana and 0.1 for the Indian Pine dataset when the proposed method was applied.

Keywords. Neural network; fuzzy systems; neuro-fuzzy systems; hyperspectral image classification.

\section{Introduction}

Hyperspectral images, containing hundreds of adjacent spectral bands, are considered to be a rich source, which can help to detect and discriminate between specific phenomena on earth. However, obtaining useful information and the processing of a large volume of data represent significant challenges in remote sensing. Traditional statistical classifiers, such as maximum likelihood (Richards 2013), are not capable of obtaining efficient results in the case of hyperspectral image classification, mainly due to the fact that the number of the required training samples increases in proportion to the high number of input data dimensions. This is referred to as the Hughes phenomenon (Hughes 1968) in the literature. The lack of sufficient training samples causes a singularity in most of the statistical processing methods.

However, non-statistical methods, which mainly focus on the study of spectral patterns in detail, are widely used for hyperspectral data interpretation. Examples include spectral angle mapper (SAM) or multiple endmember spectral mixture analysis (Dennison and Roberts 2003; Dennison et al. 2004; Song 2005; Franke et al. 2009; Somers et al. 2011; 
Petropoulos et al. 2015) methods. These methods are highly dependent on the exact determination of pure pixels or endmembers. However, endmember extraction represents another challenge, due to the presence of mixed pixels in a large number of pixels in satellite images.

Considering the above-mentioned problems, a new approach is to apply artificial intelligence (AI) for hyperspectral data interpretation. AIbased methods are applied in an attempt to solve the problems encountered by traditional tools for classification of complex hyperspectral images. The two best-known AI-based methods are neural networks and fuzzy systems (Torabi et al. 2015).

The most important characteristic of neural networks is their ability to simulate the learning capabilities of human beings in order to use these capabilities for future decision making (Braspenning and Thuijsman 1995; Mehrotro et al. 2000). For classification purposes, neural networks can learn from the available training samples and can apply this knowledge to the remaining samples in order to give them a meaningful class label.

Neural networks have been used to classify multispectral and hyperspectral images in some previous studies. For example, in Zhang and Xie (2012), a neural network was designed to produce an accurate vegetation map from hyperspectral images. A semi-supervised neural network was designed for hyperspectral classification in Ratle et al. (2010). In Chi and Ersoy (2005) a new learning system, called a statistical self-organising learning system, was introduced for remote sensing applications. In Jimenez et al. (1999), researchers used data fusion techniques to analyse the high-dimensional data. In addition, in Arslan (2009), the qualification of neural networks used in image classification was investigated via a statistical method.

Although neural networks are used in this area, their most critical problem is that they are black boxes. This is a significant weakness, for without the ability to produce comprehensible decisions it is hard to trust the reliability of networks addressing real-world problems (Benítez et al. 1997). In other words, since there is no semantic relationship between the final answer and the internal parameters of neural networks, it is hard to improve their operation.

Fuzzy logic, as another AI-based tool, tries to simulate special human abilities in making true decisions or in arguing from inadequate and inaccurate information. Fuzzy logic generates systems that apply the knowledge of an expert to produce appropriate rules in order to make suitable decisions.

Many studies have been undertaken in hyperspectral data analysis based on the special capabilities of fuzzy systems. In Yamany et al. (1999), a fuzzy classifier was designed for automating target detection in hyperspectral images. In Kuo et al. (2008), a new method called fuzzy c-weighted means was proposed for hyperspectral image clustering. In Bilgin et al. (2008), fuzzy approaches were applied to exploit membership relations based on spatial information and to design an unsupervised classifier. Other studies can be found in Borasca et al. (2006), Picón et al. (2009) and Alajlan et al. (2012).

Fuzzy systems also have their shortcomings. They cannot be trained over the existing samples. They can only arrange the expert knowledge in the form of if-then rules, and if some aspects of the subject are ignored deliberately or inadvertently by experts, the system does not work well and can make the task even more complicated. Furthermore, defining the appropriate membership functions/values for each parameter is a challenging task.

According to the above discussion, it seems that fuzzy systems and neural networks could be integrated to avoid their deficiencies and improve their capabilities. This leads to new systems called neuro-fuzzy systems. A neuro-fuzzy system is basically a fuzzy system that uses a learning algorithm, derived from or inspired by neural network theory, to determine its parameters (fuzzy sets and fuzzy rules) based on sample data (Nauck et al. 1997).

The definition expresses the fact that neural networks can help fuzzy systems to obtain the required information for rule generation, and fuzzy systems open the black box of neural networks, enabling the users to follow each step of the learning process and to comprehend the steps easily in order to apply any further corrections.

A new neuro-fuzzy system for remote sensing data classification was proposed by Qiu and Jensen (2004), which is, in fact, Kohonen's LVQ2, fuzzified using Gaussian membership functions. This system, which is called Gaussian fuzzy learning vector quantisation (GFLVQ), was later developed to effectively classify hyperspectral images in Qiu (2008). GFLVQ takes advantage of the unsupervised learning algorithm of self-organising map (SOM), while at the same time the supervised learning capabilities of LVQ are exploited. 
Although GFLVQ can obtain satisfactory results, several problems arise in cases of complicated images where more detailed classes are required. Such problems can be due to the simple parameterisation of GFLVQ or due to the lack of any preprocessing.

In this paper, the aim is to design a novel neuro-fuzzy system for detailed classification of hyperspectral images of both rural and urban areas. This neuro-fuzzy system is fundamentally based on GFLVQ but differs in some respects. First, a preprocessing step is added to the neurofuzzy system which can help improve the efficiency of the system by normalising and reducing the data and facilitating the processes of the training phase. Second, a simple change in the training procedure is made, in order to increase the final classification accuracy. The proposed neuro-fuzzy system is then used to classify three different datasets: (i) a hyperspectral image of Pavia University showing an urban area with nine different classes, from the reflective optics system imaging spectrometer (ROSIS) sensor with a spatial resolution of $1.3 \mathrm{~m}$, (ii) a Botswana image of a rural area with 14 different classes from Hyperion with a spatial resolution of $30 \mathrm{~m}$ and (iii) the Indian Pine dataset with 10 natural and agricultural classes from an airborne visible/infrared imaging spectrometer (AVIRIS) sensor with a spatial resolution of 1-4 m. These three datasets were chosen to test the system.

As a neural network, the system needs to receive a sufficient number of training samples to be trained well. Therefore, the number of training samples required and the number of bands required for each dataset have been calculated. In order to test the generalisation of the system, each experiment was repeated 50 times and the means of the results were used. Final results show that our proposed system achieved high accuracy and outperforms some common classification methods.

In the following, first the neuro-fuzzy classifier is introduced along with its required preprocessing steps. Then, the datasets are introduced. In the next part, the results are given and discussed, and the final section is attributed to a conclusion.

\section{Methodology}

In this section, the detailed methodology of the desired system is first explained step by step. Then, the principles of classification accuracy assessment methods are explained.

\subsection{Proposed method}

The proposed method is described in four subsections, i.e., data preparation, neuro-fuzzy system design, system learning and system recalling.

\subsubsection{Data preparation}

Hyperspectral images, with a dense sampling of wavelengths, consist of a large number (tens or hundreds) of spectral bands. This quantity of input data imposes great computational effort. In addition, the redundancy caused by the high correlation between these spectral bands may result in the failure of the system training stage. Accordingly, data reduction is a common practice in hyperspectral data analysis.

In this research, we used two-stage preprocessing, which was inspired by the initial idea introduced in Aghaee and Mokhtarzade (2015). This preprocessing has the ability to compress data based on the information of each class. Therefore, it speeds up the process of learning in the training phase of the neuro-fuzzy system. To apply this preprocessing, band variances are first considered, in order to normalise the input training samples. Subsequently, the principal component analysis (PCA) technique (Jolliffe 2002) is applied individually on each set of training samples. This kind of data reduction has a great impact on the success of the following learning stage of the neuro-fuzzy system and makes the system capable of image classification in detail.

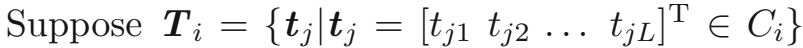
is the set of training samples belonging to a class $C_{i}(i=1,2, \ldots, m)$, where $j=1,2, \ldots, n_{i}$ is the number of training samples and $L$ shows the input data dimension (number of spectral bands). The variance-based normalisation is as follows:

$$
\begin{aligned}
& \boldsymbol{N}=\operatorname{diag}\left[\frac{1}{\sigma_{i 1}^{2}} \frac{1}{\sigma_{i 2}^{2}} \cdots \frac{1}{\sigma_{i L}^{2}}\right], \\
& \boldsymbol{t}_{j}^{\text {normalised }}=\boldsymbol{N}_{i} \cdot \boldsymbol{t}_{j},
\end{aligned}
$$

where $\sigma_{i k}^{2}$ is the variance of the $k$ th band computed over the training samples of class $C_{i}$ (i.e., $T_{i}$ ). In this normalisation, the training samples of each class are compressed based on their variances. Therefore, the unjustified high influence of wide bands and too-distant samples are controlled at the learning stage of the system. 
Regarding this distinct normalisation of different target classes, each class is individually introduced to the PCA data reduction (equation 2):

$$
\boldsymbol{T}_{i}^{*}=\left\{\boldsymbol{t}_{j}^{*} \mid \boldsymbol{t}_{j}^{*}=\boldsymbol{B}_{i} \cdot \boldsymbol{t}_{j}^{\text {normalised }}\right\} .
$$

In this equation, $\boldsymbol{B}_{i}$ is the coefficient matrix reduced via $\mathrm{PCA}$ which is computed over the normalised training samples of class $C_{i}$. The complete flowchart showing the data preparation process is presented in figure 1.

\subsubsection{Neuro-fuzzy system design}

The theory underlying our proposed method is based on a special kind of neuro-fuzzy system called a fuzzy self-organising map (FSOM), which was first introduced in Nomura and Miyoshi (1995) and $\mathrm{Su}$ and Tew (2000).

This system is actually a fuzzified SOM (Kohonen 1990) where the learning results are presented as a number of fuzzy rules which are extracted adaptively from the training samples. Figure 2 shows the structure of the applied neurofuzzy system.

Considering $\boldsymbol{x}=\left[x_{1}, \ldots, x_{n}\right]^{\mathrm{T}} \in R^{n}$ as the input vector and the $i$ th neuron in the system $(i=1,2, \ldots, m)$, each input component is fuzzified via a Gaussian membership function $F_{i j}$, which is designed to occur between the $j$ th input component and the $i$ th neuron (equation 3 ):

$$
\begin{array}{r}
F_{i j}(\underline{x})=\exp \left((-1 / 2)\left(c_{i j}-x_{j}\right)^{2} / \sigma_{i j}^{2}\right), \\
(i=1, \ldots, m, j=1, \ldots, n) .
\end{array}
$$

According to equation (3), a centre vector $c_{i}=$ $\left[c_{i 1}, \ldots, c_{i n}\right]^{\mathrm{T}}$ and a standard deviation vector $\boldsymbol{\sigma}_{i}=$ $\left[\sigma_{i 1}, \ldots, \sigma_{i n}\right]^{\mathrm{T}}$ are assigned to each neuron for fuzzification. The fuzzified input components are applied to determine the neuron outputs $F_{i}(x)$ $(i=1,2, \ldots, m)$.

Multiplication, min or max operators are commonly applied to compute the neuron outputs (i.e., $F_{i}(\underline{x})$ ). In Nomura and Miyoshi (1995), the multiplication operator is applied, although in Qiu and Jensen (2004), this operator is criticised, as it highly reduces the output values. The $\min / \max$ operators give more reasonable output values; however, these operators select one of the input parameters and ignore the numerical values of the rest.

Inspired by Qiu (2008), in this research the andor fuzzy operator is applied to compute the neuron outputs. This operator is actually a geometrical

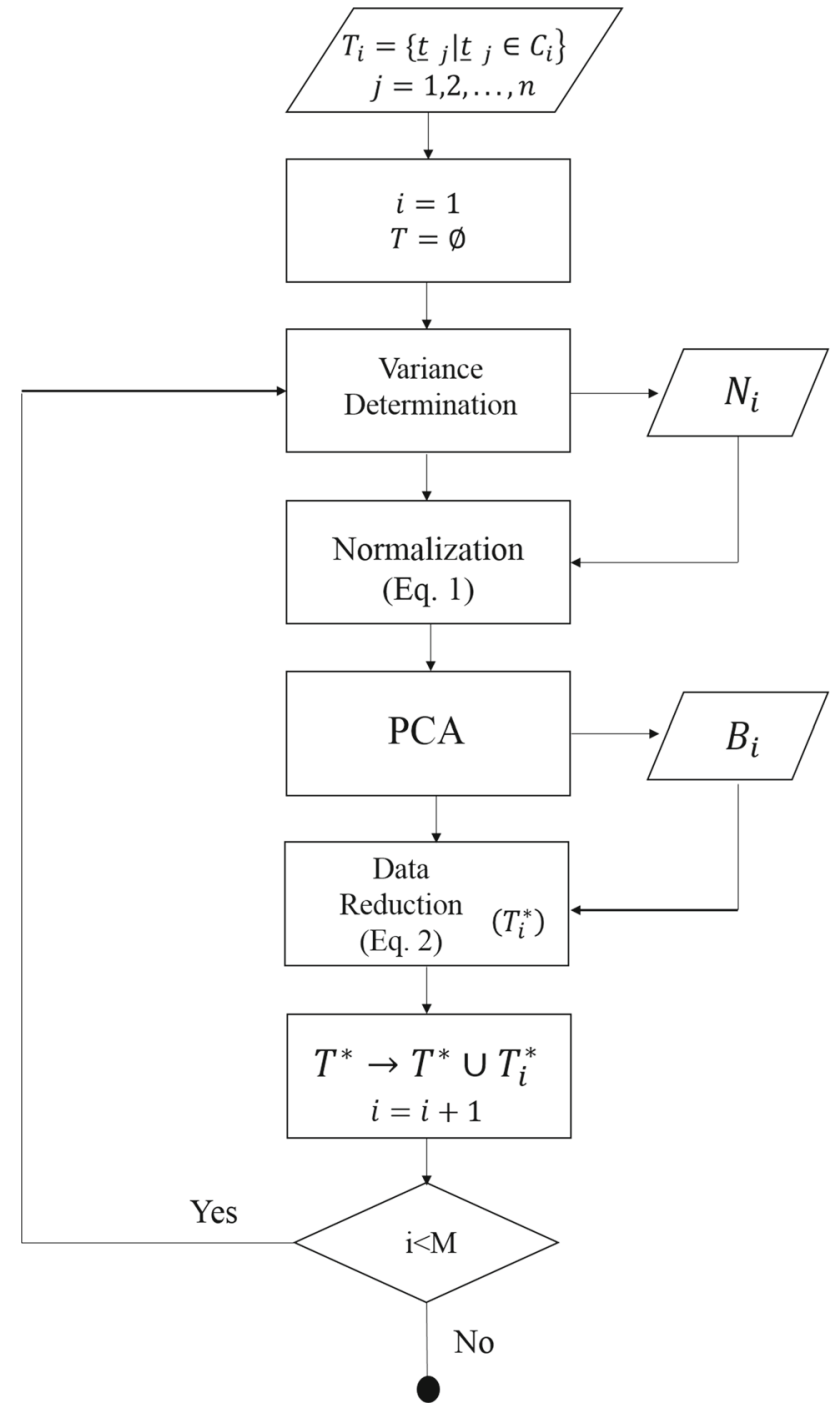

Figure 1. Flowchart of data preparation.

mean computed over the fuzzified input parameters (equation 4):

$$
\begin{aligned}
\boldsymbol{F}_{i}(x) & =\left[\prod_{j=1}^{n} F_{i j}(x)\right]^{1 / n} \\
& =\exp \left(\frac{1}{n} \sum_{j=1}^{n}-(1 / 2) \frac{\left(c_{i j}-x_{j}\right)^{2}}{\sigma_{i j}^{2}}\right) .
\end{aligned}
$$

The fuzzy and-or operator of equation (4) stands somewhere between the fuzzy-and (intersection) and the fuzzy-or (union) operators. This operator can compensate for the low numerical values of some input parameters with higher values for the others. This characteristic can overcome the noisy behaviour of the input parameters, which represents a challenge in hyperspectral data analysis. The computational procedure mentioned for each 


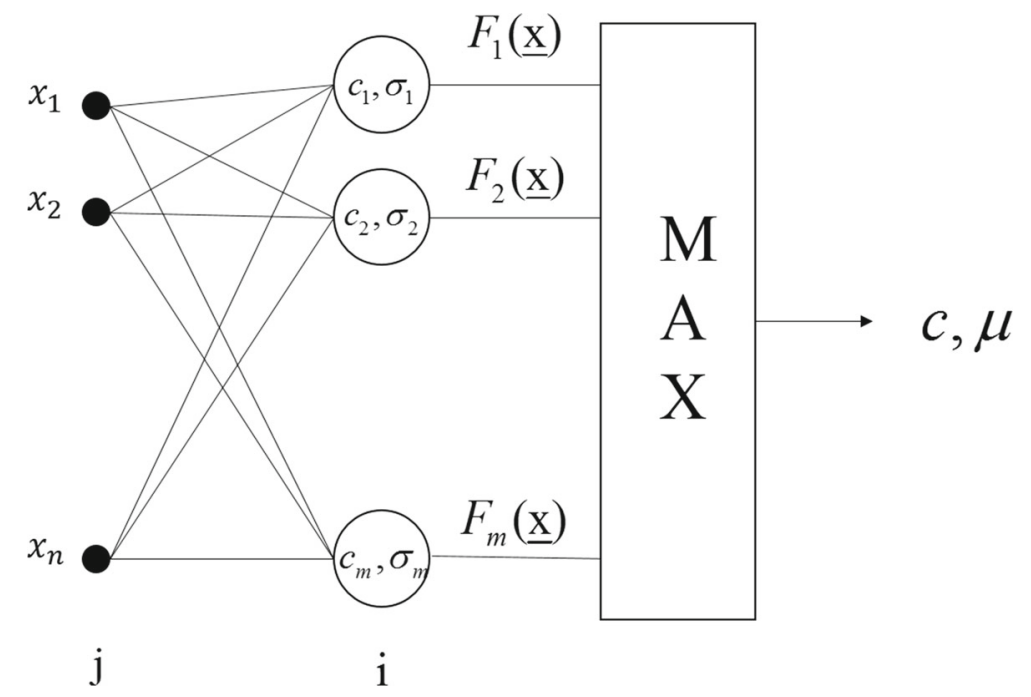

Figure 2. The structure of the applied neuro-fuzzy system.

system neuron can be regarded as applying the following fuzzy rule:

IF band1 has membership value $F_{i 1}$ and/or band 2 has membership value $F_{i 2}$ and-or ... and-or band $n$ has membership value $F_{i n}$, then the pixel $\underline{x}$ belongs to class $i$ with membership value $F_{i}$.

These fuzzy rules indicate that an individual neuron should be devoted to each target class and thus, for classification purposes, the number of neurons in FSOM is equal to the number of classes. In this way, each input sample will contain the membership values for all target classes, where the higher value determines the final response of the network (equation 5):

$$
\left\{\begin{array}{l}
c=\underset{i}{\arg \max } F_{i}(x), \\
\mu=F_{c}(x) .
\end{array}\right.
$$

In this equation, $c$ is the number of the selected target classes and $\mu$ is the membership value assigned to the input sample. Notice that in SOM, the winner neuron is the one with the minimum distance from its centre to the input sample. However, in FSOM, the winner neuron is chosen according to the maximum membership value.

In Nomura and Miyoshi (1995), the fuzzy rules of the neurons are weighted; however, in this research, all the classes are regarded as of the same importance and no weighting is applied.

\subsubsection{System learning}

As in traditional SOM, competitive learning is also applied to the training of FSOM. However, in SOM, only the centre parameters of neurons are trained, while standard deviation vectors also contribute to the training process of FSOM.

In the learning system of FSOM, the centre and standard deviation vectors of the winner neuron (corresponding to the target class with maximum membership value) are adjusted to minimise the following cost functions:

$$
\left\{\begin{array}{c}
E_{1}\left(x, c_{i}\right)=(1 / 2) \sum_{j=1, \ldots, n}\left(x_{j}-c_{i j}\right)^{2} \quad \forall x \in \mathbf{C}_{i}, \\
E_{2}\left(x, \sigma_{i}\right)=(1 / 2) \sum_{j=1, \ldots, n}\left(\sigma_{i j}^{2}-\left(x_{j}-c_{i j}\right)^{2}\right)^{2} \\
\forall x \in \mathbf{C}_{i} .
\end{array}\right.
$$

The following learning rules are obtained by taking the derivative of the cost functions and applying the gradient descent technique:

$$
\begin{aligned}
& \Delta c_{i j}= \begin{cases}\lambda\left(x_{j}-c_{i j}\right) & \text { if } x \in C_{i}, \\
0 & \text { otherwise, }\end{cases} \\
& \Delta \sigma_{i j}= \begin{cases}2 \lambda \sigma_{i j}\left(\left(x_{j}-c_{i j}\right)^{2}-\sigma_{i j}^{2}\right) & \text { if } x \in C_{i}, \\
0 & \text { otherwise. }\end{cases}
\end{aligned}
$$

In these equations, $\lambda \in[0,1]$ is the learning rate and equation (7) is same as the learning equations in SOM, moving the centre of the neuron towards the centroid of its corresponding class samples. However, equation (8), especially in FSOM, adjusts the standard deviation vectors according to the dispersion of the target classes. In this way, 
the more compact classes are moved restrictively to assign membership values to those samples at the same distance from their centres.

In Qiu (2008), it was reported that the learning process of equation (8) is not applicable to remote sensing data as it causes the divergence of the system, and therefore an alternative equation was proposed. It was also reported that data normalisation cannot solve the problem. However, in the present study, no divergence occurred. This was due to the data preparation as described in section 2.1.1. The data preparation procedure removes the high correlations usually seen in remote sensing data, via PCA. Therefore, rather than the procedure introduced by Qiu (2008), we use equation (8) to adjust the standard deviation.

In the learning process of the SOM, it is recommended to add a punishment term to the neurons which are wrongly selected as the winner (i.e., when the input training sample does not belong to the corresponding target class of the winner neuron). If the $i$ th neuron is selected as the winner, the revised learning rule for the centre parameters is as shown in equation (9):

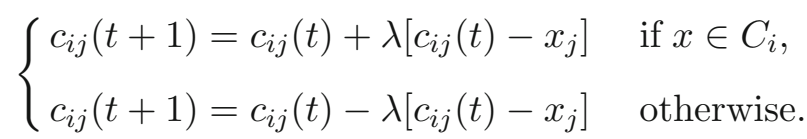

In this learning process, the centre parameters of the winner neuron are adjusted to both correct and incorrect classification of the input training samples. In other words, this updating rule moves the centre vectors towards the correctly matched training samples and away from the wrong one(s). This modification can greatly improve the learning speed.

For network initialisation (initial rules for the centre and the standard deviation vectors of each neuron), the centre and the standard deviations of the training sample sets are used. This method has been used previously in Zhang and Foody (2001) to obtain the centre of the classes. Here, as recommended in Qiu (2008), it is merely used to initialise the network.

\subsubsection{System recalling}

As mentioned in previous sections, system learning is performed using the training sets of the different classes, where each set is prepared (normalised and reduced via PCA) with different parameters.

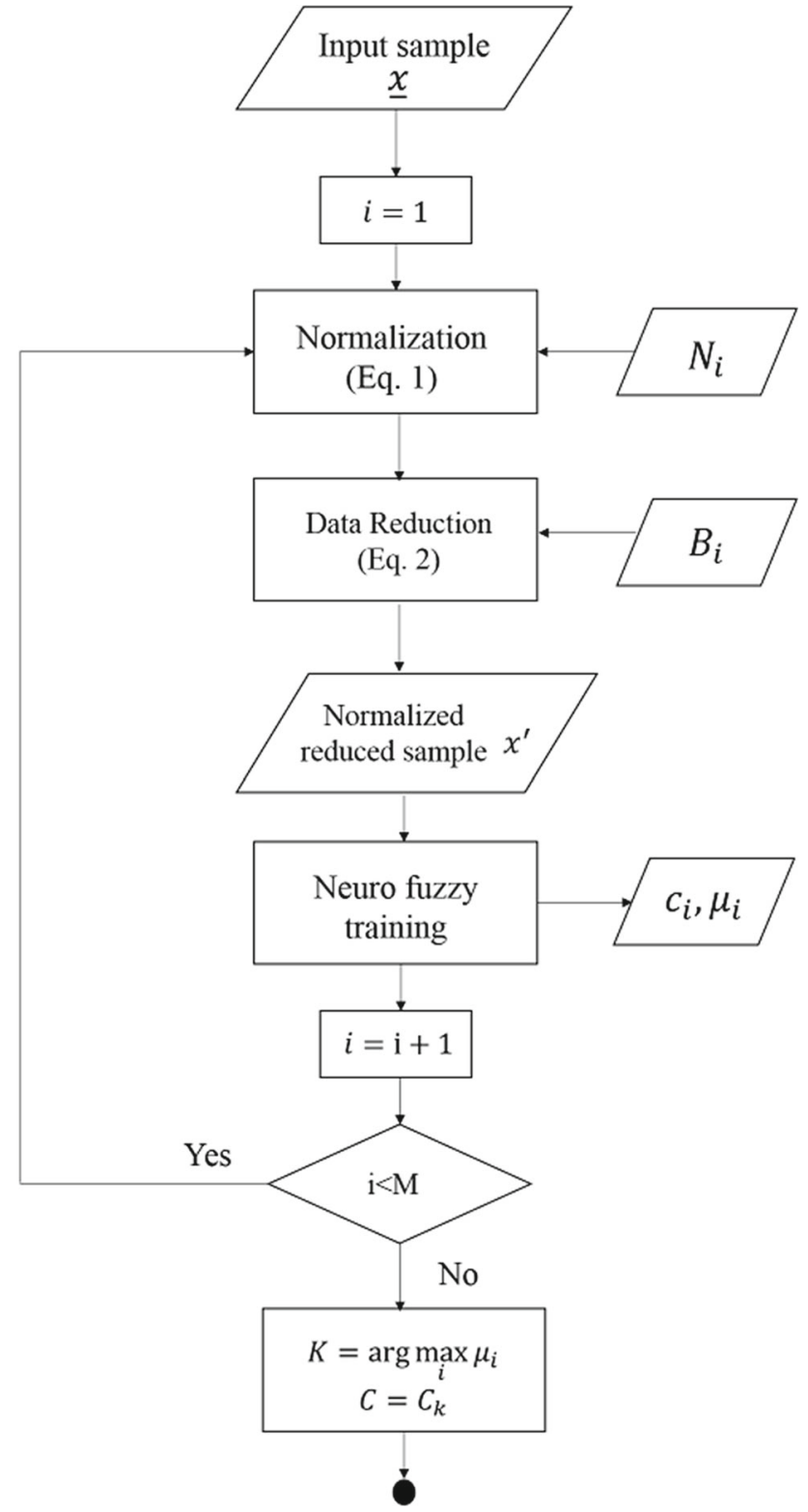

Figure 3. System recalling flowchart.

Accordingly, a separate system recalling process should be followed for each target class. This way, each new input sample is normalised and reduced via $\mathrm{PCA}$ with the preparation parameters of all target classes, and then presented to the network. Finally, the input sample is assigned to the class with the maximum membership value. The system recalling flowchart is presented in figure 3 .

In figure $3, c$ shows the class number for the input sample.

\subsection{Different metrics for accuracy assessment}

The accuracy assessment is necessary to allow confidence to be attached to the final results and it 
will serve to indicate whether the objectives of the analysis have been achieved.

There are several ways of assessing the accuracy of a thematic map, and there are several measures available for expressing that accuracy. One of them is the $\kappa$ coefficient. The $\kappa$ coefficient is a measure of classifier performance derived from the confusion (error) matrix but which, purportedly, is free of any bias resulting from a chance agreement between the classifier output and the reference data (Richards 2013).

However, there is now significant concern that the $\kappa$ coefficient is a misleading measure (Pontius and Millones 2011). Five different $\kappa$ indices are examined in Pontius and Millones (2011), and it is concluded that the $\kappa$ indices are useless, misleading and/or flawed for practical applications in remote sensing. It is recommended that instead of using the $\kappa$ coefficient, professionals should summarise the confusion matrix with two simpler summary parameters: quantity disagreement and allocation disagreement. The allocation disagreement is defined as the amount of difference between the reference map and a comparison map which is a less-than-optimal match in the spatial allocation of the categories, with respect to the available categories in the reference and comparison maps. The quantity disagreement is defined as the amount of difference between the reference map and a comparison map which is a less-than-perfect match with respect to the available categories in the reference and comparison maps. To derive these measures, equation (10) can be written as

$$
p_{+i}=\sum_{k=1}^{M} p_{k i}, \quad p_{i+}=\sum_{k=1}^{M} p_{i k},
$$

where $p_{i j}$ represents the confusion matrix entries expressed as proportions or probabilities. The term $p_{i j}$ is a short hand for the joint occurrence $p(m=$ $i, r=j$ ) when the relative class proportions in the reference data reflect the true proportions on the ground. Taking the difference between $p_{+i}$ and $p_{i+}$ is tantamount to taking the difference between the number of class $i$ pixels in the reference data and the number in the thematic map. Summing the absolute difference over all classes gives the discrepancy in the proportions of all classes between the reference data and the map:

$$
Q=1 / 2 \sum_{i=1}^{M}\left|p_{+i}-p_{i+}\right|
$$

Here, $Q$ is called the quantity disagreement (equation 11). Another interpretation is that it effectively measures the differences in the areas allocated to the classes in the reference data and the map. This is one type of error measure. A second error measure is the allocation disagreement defined by

$$
A=\sum_{i=1}^{M} \min \left\{\left(p_{+i}-p_{i i}\right),\left(p_{i+}-p_{i i}\right)\right\} .
$$

This measure is intended to assess the aggregated misallocation of individual pixels for the same level of quantity agreement. If there is a specific error of commission there is a corresponding error of omission, which is recognised in the minimum operation of equation (12). If we represent an entry in the error matrix by $n_{i j}$ and the total number of pixels by $N$, then the probability of correct classification is $p_{0}$ as defined in equation (13):

$$
p_{0}=\frac{1}{N} \sum_{i=1}^{M} n_{i i}
$$

The total error or disagreement between the thematic map and the reference data is $1-p_{0}$. Interestingly, it can be shown that

$$
1-p_{0}=A+Q
$$

indicating that the total disagreement can be disaggregated into quantity and allocation disagreements.

The accuracy assessment is necessary to allow confidence in the final results and it will serve to indicate whether the objectives of the analysis have been achieved.

\section{Datasets and case studies}

Three different input images are used in this study. The first one is the space-born hyperspectral image from the Hyperion sensor, taken over a rural area. The second is the airborne hyperspectral image from the ROSIS sensor taken over an urban area. The last is an aerial image from AVIRIS which contains both natural and agricultural classes. These images are introduced in the following sections. 


\subsection{Satellite image from rural area, Okavango Delta, Botswana}

The NASA EO-1 satellite acquired a sequence of data over the Okavango Delta, Botswana in 2001-2004. The Hyperion sensor on EO-1 acquires data at a pixel resolution of $30 \mathrm{~m}$ over a 7.7$\mathrm{km}$ strip in 242 bands covering the $400-2500 \mathrm{~nm}$ portion of the spectrum in 10-nm windows. Hyperion is a push-broom imager with 12-bit image data, $6 \%$ absolute radiometric accuracy, image swathe width of $7.5 \mathrm{~km}$ and temporal resolution of 200 days. Preprocessing of the data was performed by the University of Texas Center for Space Research to mitigate the effects of bad detectors, interdetector anomalies, miscalibration and intermittent anomalies. Uncalibrated and noisy bands that cover water absorption features were removed, and the remaining 145 bands were included as candidate features: [10-55, 82-97, 102-119, 134-164, 187-220]. The data analysed in this study, acquired on 31 May 2001, consisted of observations from 14 identified classes representing the land cover types in seasonal swamps, occasional swamps and drier woodlands located in the distal portion of the delta (Neuenschwander et al. 2005). These classes were chosen to reflect the impact of flooding on vegetation.

These data consist of observations from 14 identified classes representing the land cover types located in the distal portion of the delta (Neuenschwander et al. 2005). They include: Water, Hippo grass, Floodplain grass1, Floodplain grass2, Reeds1, Riparian, Firescar2, Island interior, Acacia woodlands, Acacia shrub lands, Acacia grasslands, Short mopani, Mixed mopani and Exposed soils. Classes 3 and 4 are both floodplain grasses that are seasonally inundated but differ in their hydroperiod (the amount of time inundated). Classes 9-11 represent different mixtures of acacia woodlands, shrublands and grasslands and are named according to the dominant class. Reference data, including test and train data, were selected manually using a combination of global positioning system-located vegetation surveys, aerial photography from the Aquarap (2000) project and 2.6-m resolution IKONOS multispectral imagery (Chi and Ersoy 2005).

\subsection{Aerial image from urban area}

For the airborne hyperspectral image, an image recorded by the ROSIS optical sensor over the urban area of Pavia University, Italy, was used. The image was taken by the Deutsches Zentrum für Luft- und Raumfahrt (DLR, the German Aerospace Agency) in the framework of the HYSENS project, and is $610 \times 340$ pixels, with a spatial resolution of $1.3 \mathrm{~m} /$ pixel. The ROSIS is designed to cover a spectral range from 430 to $960 \mathrm{~nm}$ in resolution steps of $5 \mathrm{~nm}$ per channel. The radiometric resolution is defined as $0.05 \%$ of the apparent albedo. The total field of view (FOV) covers $+16^{\circ}$ per optics module.

The number of data channels in the acquired image is 115 (with a spectral range from 0.43 to $0.86 \mu \mathrm{m}$ ). The 12 noisiest channels were removed and the remaining 103 bands were used for the experiments. Training data consisted of observations from nine different classes of available objects in the scene. The target classes were: Asphalt, Meadows, Gravel, Trees, Painted metal sheets, Bare soil, Bitumen, Self-blocking bricks and Shadows.

\subsection{Aerial image with natural and agricultural classes}

The third dataset is a subimage from AVIRIS with a size of $145 \times 145$ pixels, taken over northwest Indiana's Indian Pine test site in June 1992. This dataset has 16 classes. The sensor is a whiskbroom system utilising scanning foreoptics to acquire cross-track data. The instantaneous field of view (IFOV) is $1 \mathrm{mrad}$. The total scan angle is $30^{\circ}$ and all data are stored in 12-bit integers. The temporal resolution for this sensor is on demand.

The Indian Pine scene is two-thirds agricultural and one-third forest or other natural perennial vegetation (Mojaradi et al. 2009). There are two major dual-lane highways and a railway line, as well as some low-density housing, other built structures and smaller roads. Since the image was taken in June, some of the crops present, e.g., corn and soybeans, were in the early stages of growth, with less than $5 \%$ coverage. The data are distributed over 220 spectral bands, about $10 \mathrm{~nm}$ apart between 0.4 and $2.45 \mu \mathrm{m}$, with a spatial resolution of $20 \mathrm{~m}$. The 20 water absorption channels were removed from the original image. Since some classes are too small to retain enough disjoint samples for training and testing, six classes were neglected, leaving 10 classes for the experiments: Corn - no till, Corn - min, Grass/pasture, 

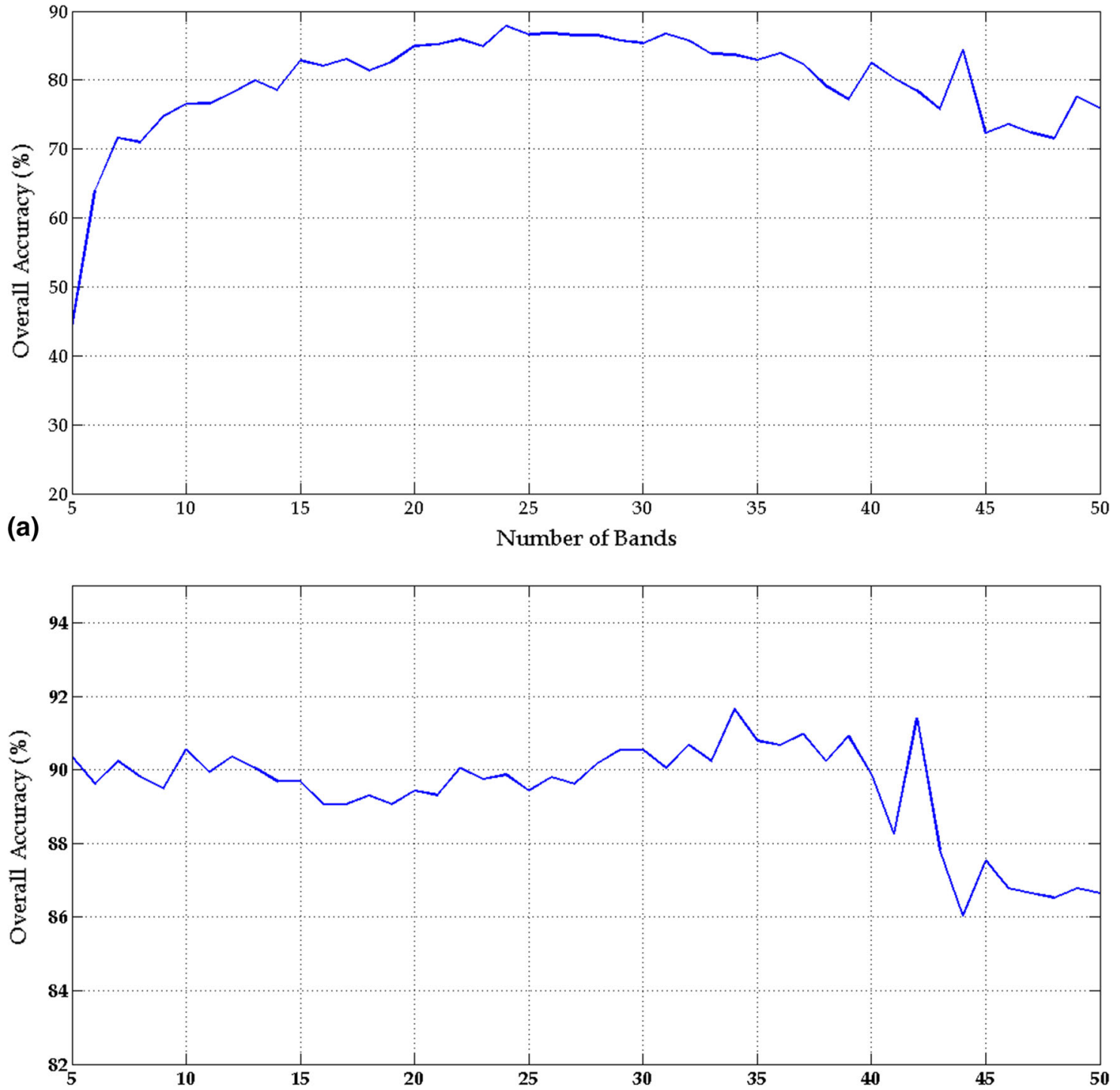

(b)

Number of Bands

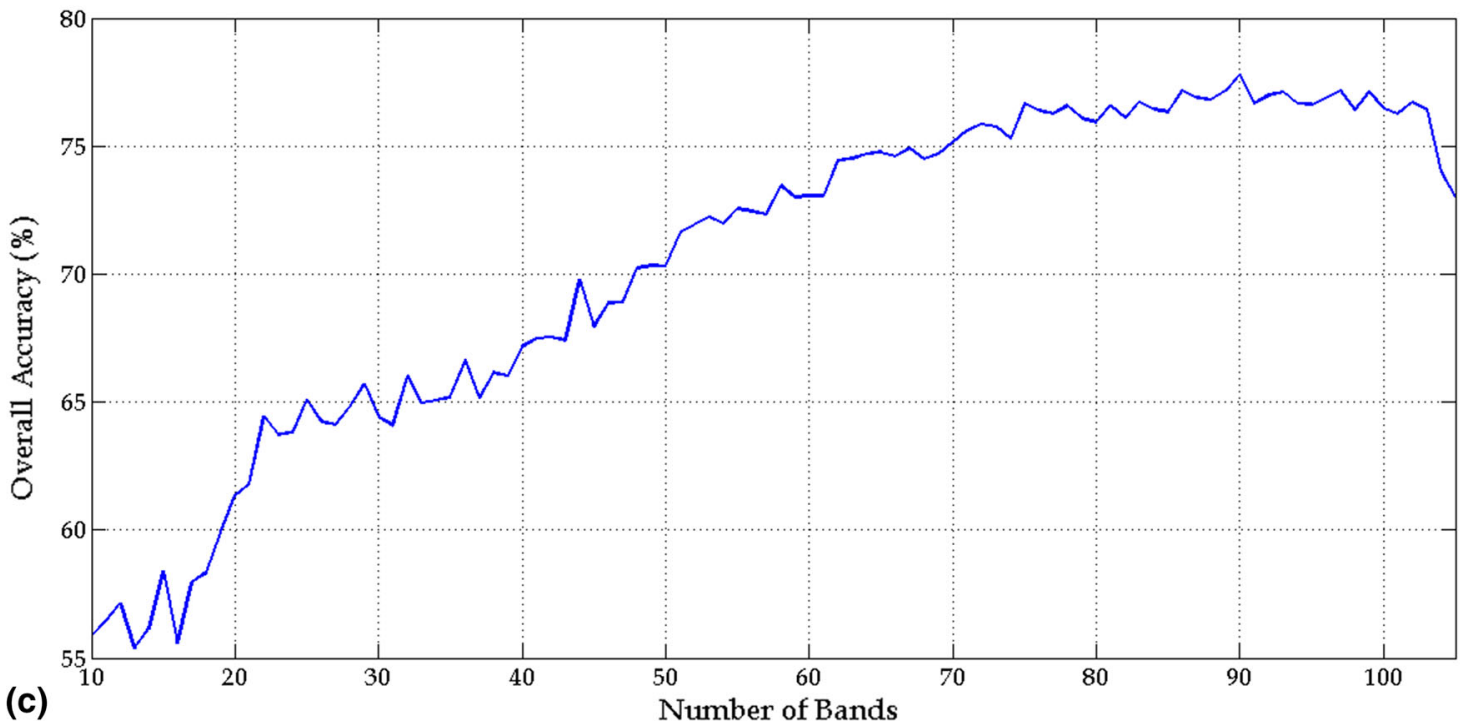

Figure 4. (a) Overall accuracy (\%) per different number of PCA bands for Pavia University image, (b) overall accuracy (\%) per different number of PCA bands for Botswana image, (c) overall accuracy (\%) per different number of PCA bands for Indian Pine image. 
Table 1. Number of desired bands and overall accuracy for each dataset.

\begin{tabular}{lccr}
$\begin{array}{l}\text { Results of first } \\
\text { stage }\end{array}$ & $\begin{array}{c}\text { Pavia } \\
\text { University } \\
\text { image }\end{array}$ & $\begin{array}{c}\text { Botswana } \\
\text { image }\end{array}$ & $\begin{array}{c}\text { Indian } \\
\text { Pine }\end{array}$ \\
\hline $\begin{array}{l}\text { Number of } \\
\text { appropriate }\end{array}$ & 24 & 34 & 90 \\
$\quad$ bands & & & \\
$\begin{array}{l}\text { Kappa coefficient } \\
\text { Overall accuracy }\end{array}$ & 0.84 & 0.91 & 0.75 \\
\hline
\end{tabular}

Grass/trees, Hay - windrowed, Soybeans - no till, Soybeans - min, Soybeans - clean, Woods and Buildings/grass/trees/drives.

\section{Results and discussion}

The experimental results are presented in four parts. In the first part, the best number of input PCA bands is computed and their optimum values are determined. In the second part, the influence of the training sample is evaluated to find the size of training sample required for the learning stage. In the next part, the proposed method is implemented on the input datasets, and the results are evaluated through different metrics. In the last part, the final classification maps are compared with the results of traditional classifiers.

\subsection{The best number of PCA bands}

In this part, different numbers of PCA bands (from 5 to 50 for Pavia University and Botswana images and from 10 to 105 for the Indian Pine image) are applied to evaluate their impact on the network performance. For each number of PCA bands, $50 \%$ of all the available ground truth samples are selected randomly as the training set. Subsequently, the trained network is recalled over the remaining $50 \%$ of the ground truth samples and the overall accuracy is determined. This process was repeated 20 times to reduce the impact of training sample selection, and the final accuracy was taken as the average of all 20 runs. The obtained results are presented in figure $4(\mathrm{a}-\mathrm{c})$ for the Pavia University, Botswana and Indian Pine images, respectively.

Figure $4(\mathrm{a}-\mathrm{c})$ shows that the optimum results are obtained with 24, 34 and 90 PCA bands for
Table 2. Per cent of information explained by chosen PCA bands for each dataset.

\begin{tabular}{lc}
\hline Dataset & $\begin{array}{c}\text { Total information explained by } \\
\text { chosen PCA bands (per cent) }\end{array}$ \\
\hline Pavia University & 98.3624 \\
Botswana & 99.8221 \\
Indian Pine & 100 \\
\hline
\end{tabular}

the Pavia University (with nine target classes), Botswana (with 14 target classes) and Indian Pine (with 10 target classes) input datasets, respectively (table 1).

Note that although using PCA can cause loss of information, we control this factor in order to be sure that the loss of information does not impact very much on the final accuracy. Hence, the percentage of available information in each PCA band is calculated during each run. Table 2 shows the sum of this factor for the chosen numbers of PCA bands.

As shown in table 2, more than $98 \%$ of the information for each dataset is preserved. Therefore, we can be sure that the amount of information loss was negligible.

\subsection{The appropriate number of training samples}

All supervised neural network-based classifiers are affected by the size of training samples. To investigate the impact of this parameter on the performance of the proposed method, different sizes of training samples are applied for system learning. For each training sample size, a random selection procedure is performed over all the available ground truth samples. To make the results independent of any of other factor, this process is repeated 50 times and the averaged overall accuracy is considered. Figure $5(\mathrm{a}-\mathrm{c})$ shows the obtained results for all input images.

Figure $5(\mathrm{a}-\mathrm{c})$ indicates that at first, the system performance is improved by an increase in the training sample size. However, when the training sample size is large enough, this improvement is no longer significant. According to the obtained result, table 3 shows the optimum sizes of training samples vs. the number of target classes and PCA bands.

In order to evaluate the performance of the neuro-fuzzy system, two other parameters were calculated: the standard deviation of the $\kappa$ coefficient, 

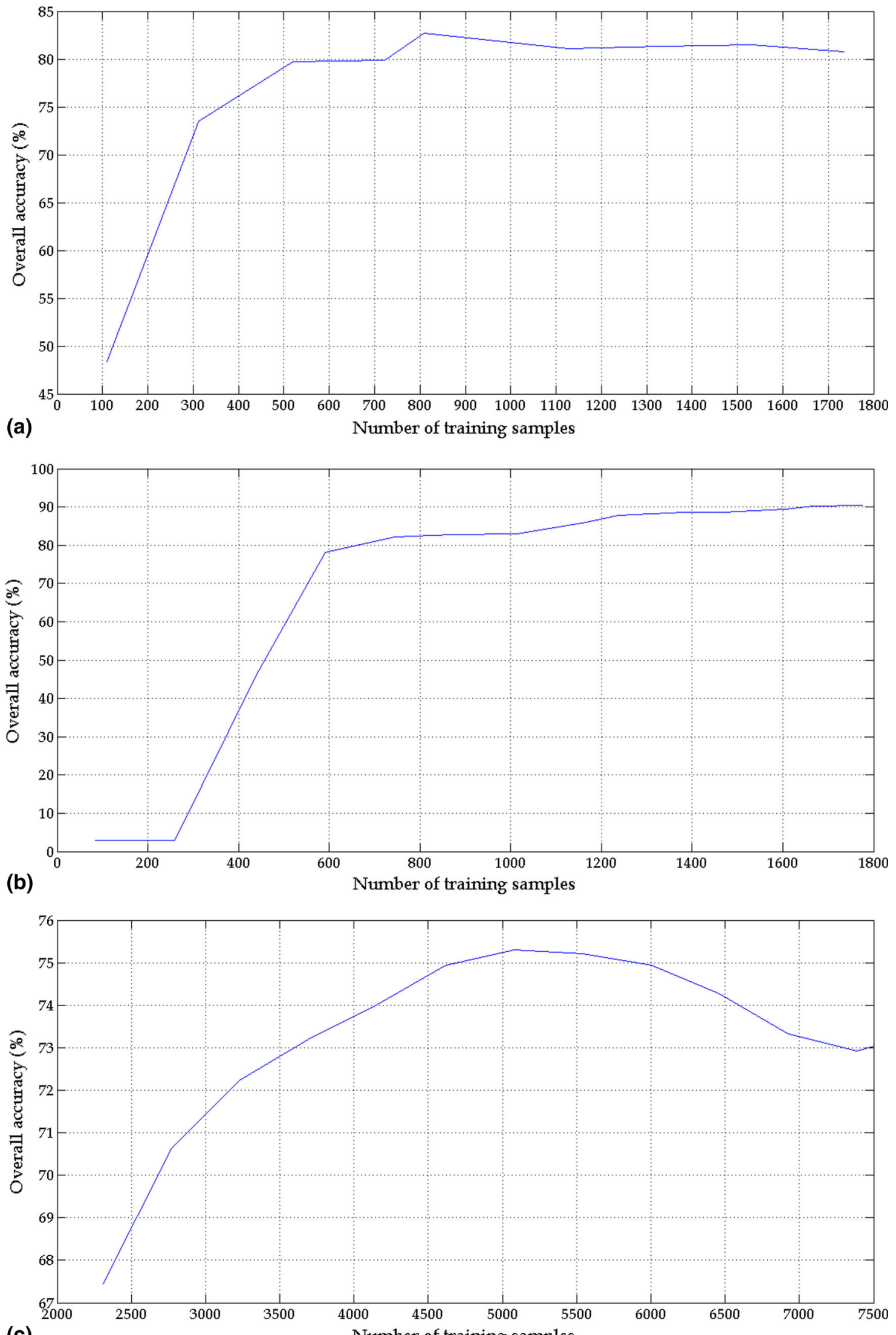

(c)

Number of training samples

Figure 5. (a) Overall accuracy (\%) via a different number of training samples for Pavia University, (b) overall accuracy (\%) via a different number of training samples for Botswana, (c) overall accuracy (\%) via a different number of training samples for Indian Pine. 
which shows the variation of this parameter over 50 repetitions, and the 'error rate', which can be computed via equation (15).

$$
\begin{aligned}
\text { Error rate }= & \text { incorrectly classified samples } / \\
& \text { classified samples. }
\end{aligned}
$$

Table 4 shows these parameters for all datasets.

Table 3. Comparison between the required parameters of the proposed system.

\begin{tabular}{lccc}
\hline Input data & $\begin{array}{c}\text { No. of target } \\
\text { classes }\end{array}$ & $\begin{array}{c}\text { No. PCA } \\
\text { bands }\end{array}$ & $\begin{array}{c}\text { Training } \\
\text { sample size }\end{array}$ \\
\hline Pavia & 9 & 24 & 809 \\
$\quad$ University & & 34 & 1653 \\
Botswana & 14 & 90 & 5078 \\
Indian Pine & 10 & \\
\hline
\end{tabular}

Table 4. Evaluation of classification via two other parameters.

\begin{tabular}{lcc}
\hline Datasets & $\begin{array}{c}\text { Standard deviation } \\
\text { of } \kappa \text { coefficient }\end{array}$ & Error rate \\
\hline Pavia University & 0.0925 & 0.2479 \\
Botswana & 0.0189 & 0.1296 \\
Indian Pine & 0.0098 & 0.2470 \\
\hline
\end{tabular}

It should be noted that these two factors refer to the mean of all experiments (not just a single run).

\subsection{Classification results}

Ground truth samples, excluding those applied for system learning, are applied for accuracy assessment of the proposed neuro-fuzzy system. Tables 5 7 show the confusion matrix for the best-trained system over the Pavia University, Botswana and Indian Pine input datasets.

The classification accuracies are evaluated using two different types of measures: the first is the $\kappa$ coefficient and overall accuracy as widely applied in remote sensing applications and the second is composed of the quantity and allocation disagreements introduced in section 2.2. These two measures were calculated for three different datasets. The total agreement measure, which is the sum of the quantity and allocation disagreements, was also computed.

It can be inferred from table 5 that class 5 (Painted metal sheets) and class 6 (Bare soil) are more mixed than the other classes. The reason may be the wide spatial distribution of these two classes, increasing the number of mixed pixels (belonging to more than one target class). Some other misclassifications, especially between class 2 (Meadows) and class 4 (Trees), are due to the high spectral similarity between those classes.

\begin{tabular}{|c|c|c|c|c|c|c|c|c|c|c|}
\hline \multicolumn{11}{|c|}{ Ground truth } \\
\hline $\begin{array}{l}\text { Classification } \\
\text { map }\end{array}$ & Asphalt & Meadows & Gravel & Trees & $\begin{array}{l}\text { Painted } \\
\text { metal sheets }\end{array}$ & $\begin{array}{c}\text { Bare } \\
\text { soil }\end{array}$ & Bitumen & $\begin{array}{c}\text { Self- } \\
\text { blocking } \\
\text { bricks }\end{array}$ & Shadows & $\begin{array}{c}\text { User } \\
\text { accuracy }\end{array}$ \\
\hline Asphalt & 5693 & 11 & 110 & 0 & 0 & 2 & 547 & 221 & 14 & 86.28 \\
\hline Meadows & 9 & 15,427 & 2 & 150 & 0 & 517 & 0 & 13 & 0 & 95.71 \\
\hline Gravel & 66 & 0 & 1350 & 0 & 0 & 0 & 6 & 349 & 0 & 76.23 \\
\hline Trees & 1 & 952 & 0 & 2686 & 0 & 36 & 0 & 0 & 0 & 73.10 \\
\hline $\begin{array}{l}\text { Painted metal } \\
\text { sheets }\end{array}$ & 216 & 11 & 25 & 6 & 1253 & 47 & 4 & 63 & 11 & 76.59 \\
\hline Bare soil & 93 & 973 & 24 & 14 & 0 & 4085 & 9 & 87 & 26 & 76.91 \\
\hline Bitumen & 22 & 0 & 0 & 0 & 0 & 0 & 669 & 0 & 0 & 96.82 \\
\hline $\begin{array}{l}\text { Self-blocking } \\
\text { bricks }\end{array}$ & 80 & 9 & 445 & 0 & 0 & 0 & 4 & 2699 & 1 & 83.35 \\
\hline Shadows & 0 & 0 & 0 & 0 & 0 & 0 & 0 & 0 & 830 & 100.00 \\
\hline $\begin{array}{l}\text { Producer } \\
\text { accuracy }\end{array}$ & 92.12 & 88.75 & 69.09 & 94.05 & 100 & 87.16 & 53.99 & 78.64 & 94.10 & \\
\hline
\end{tabular}

Table 5. Confusion matrix for Pavia University image.

Total number of test samples: 39,$868 ; \kappa$ coefficient: 0.83 ; overall accuracy: 87.02 .

Quantity disagreement: 0.05; allocation disagreement: 0.07; total disagreement: 0.12. 


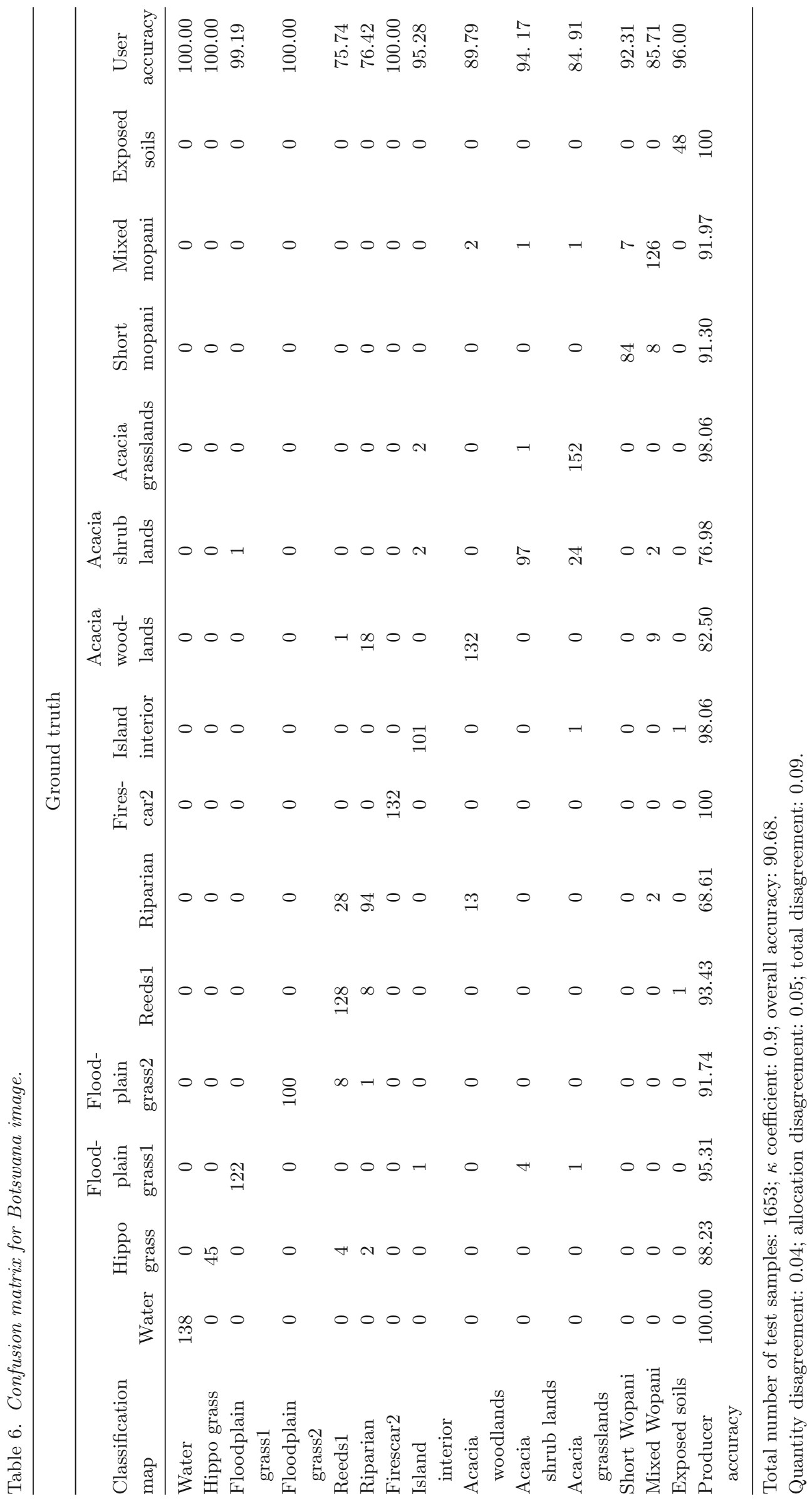




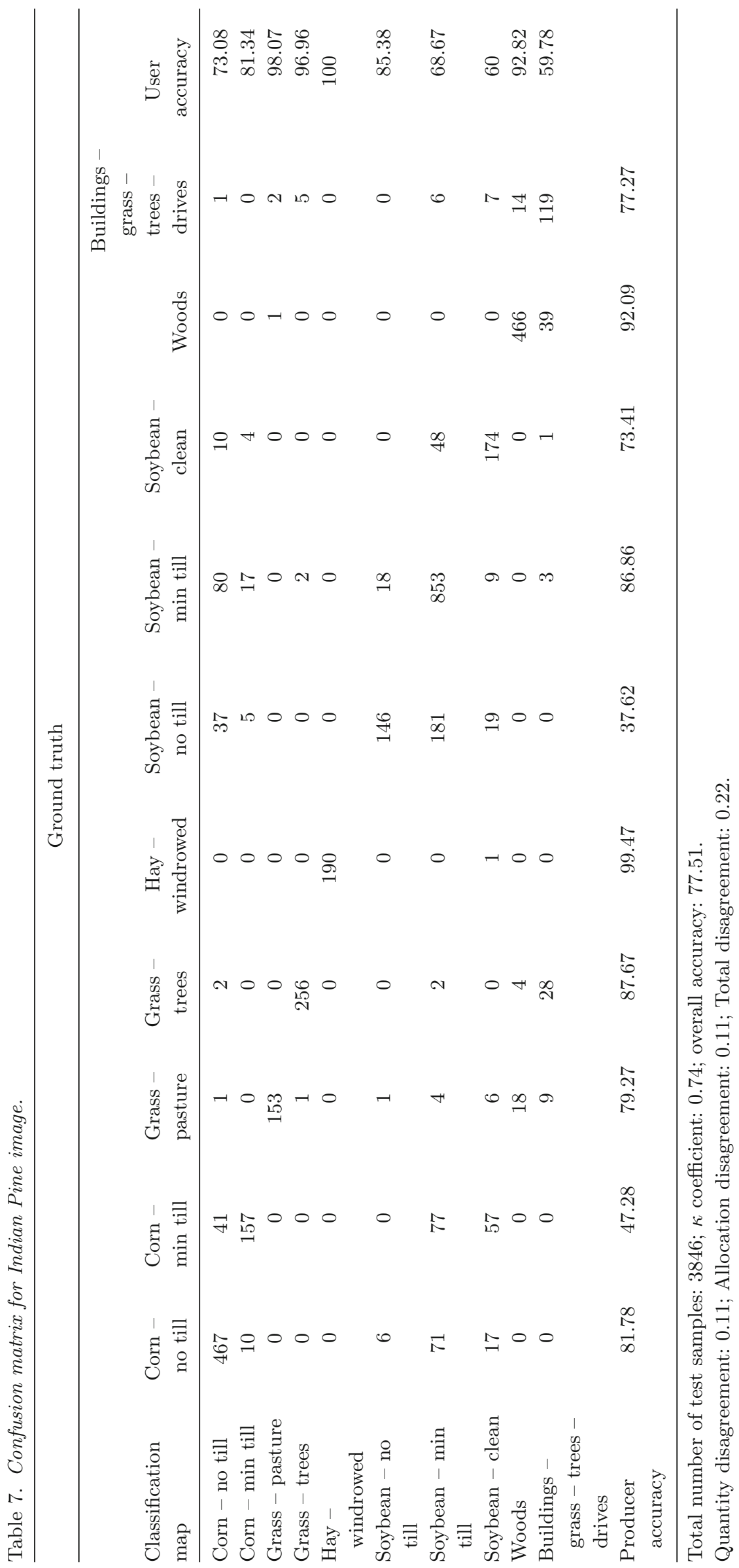


Table 8. Comparison of overall accuracy (per cent) for different classification methods.

\begin{tabular}{lccc}
\hline Method & $\begin{array}{c}\text { Pavia } \\
\text { University }\end{array}$ & Botswana & Indian Pine \\
\hline Proposed system & 87.02 & 90.68 & 77.51 \\
Traditional GFLVQ & 81.89 & 88.38 & 70.34 \\
SVM (Gaussian kernel) & 81.32 & 88.58 & 74.11 \\
SAM & 54.26 & 74.77 & 59.62 \\
\hline
\end{tabular}

Table 9. Comparison of total disagreement for different classification methods.

\begin{tabular}{lccc}
\hline Method & $\begin{array}{c}\text { Pavia } \\
\text { University }\end{array}$ & Botswana & Indian Pine \\
\hline Proposed system & 0.12 & 0.09 & 0.22 \\
Traditional GFLVQ & 0.13 & 0.12 & 0.32 \\
SVM (Gaussian kernel) & 0.13 & 0.12 & 0.23 \\
SAM & 0.35 & 0.27 & 0.37 \\
\hline
\end{tabular}

Table 5 also shows the excellent classification performance for class 9 (Shadow). Despite the fact that this class has a very limited number of training samples, the proposed system performs well (consider the user and producer accuracy for class 9$)$.

Table 6 shows that better classification results are obtained for the case of the Botswana input image. According to this table, classes 5 and 6 (i.e., Reeds1 and Riparian) are misclassified due to their mixed spatial distribution on the ground. In addition, a clear misclassification of classes 10 and 11 (Acacia woodlands and Acacia shrub lands) can be seen, which results from the very similar spectral behaviour of these classes.

Table 7 shows that the classes with spectral similarities are not classified as accurately as the others, e.g., Soybean - no till, Soybean - mini till and Soybean - clean are mixed together more than other classes. These three classes reduce the overall accuracies of the Indian Pine dataset.

It is worth noting that the $\kappa$ coefficients and the overall accuracies of this stage may be different from those in the previous stage. This is because of the change in the number and selection of training samples. In fact, both the number of PCA bands and the number of training samples affect the performance of the system directly. Therefore, in each step, we change one of them and leave the other unchanged.
Furthermore, the $\lambda$ parameter of the training part of the system is set to 0.01 in the first step and reduced linearly in each step of the learning procedure.

\subsection{Comparison with other classifiers}

Support vector machines (SVMs) have become popular for solving problems in classification, regression and novelty detection (Srivastava et al. 2012; Singh et al. 2014; Kumar et al. 2016). An important property of SVMs is that the determination of the model parameters corresponds to a convex optimisation problem (Bishop 2006).

One of the important characteristics of the SVM classifier is its ability to classify high-dimensional data with a limited number of training samples. Thus, SVM classifiers (often with kernels) are widely used in hyperspectral analysis. However, their performance depends directly on the type of kernel chosen. Therefore, they cannot be generalised to different types of data.

In this section, the proposed neuro-fuzzy system is compared with one of the most common kernel-based SVMs with a Gaussian kernel and with the so-called SAM classification method for hyperspectral images. This was done using ENVI 4.7 software and the parameters were chosen over three or four tests using the parameters suggested by ENVI itself. We also implemented the traditional GFLVQ method introduced in Qiu (2008), in order to compare it with the proposed method. 


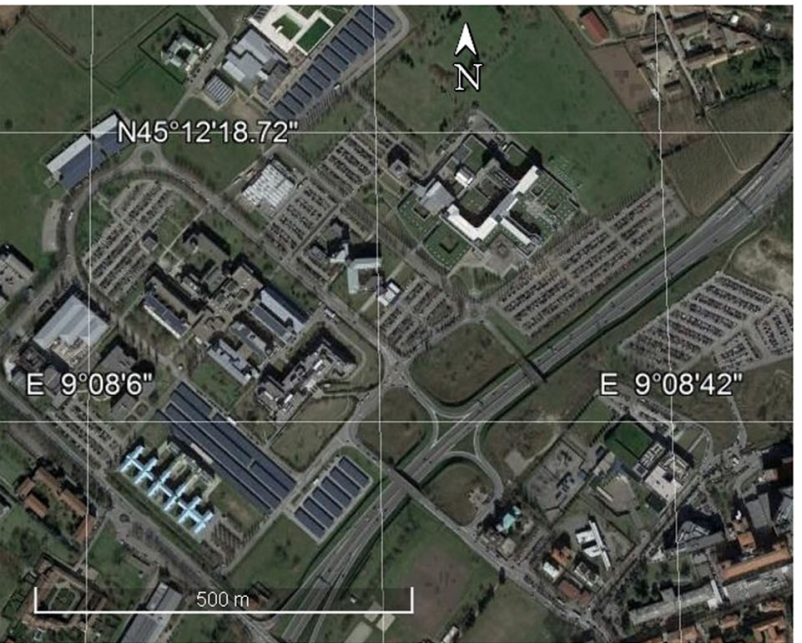

(a)

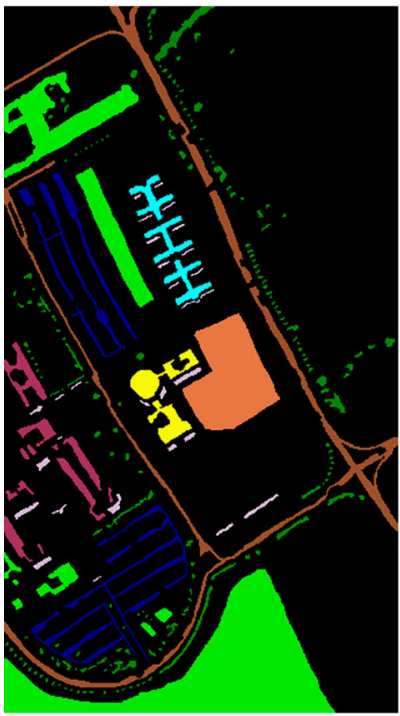

(c)

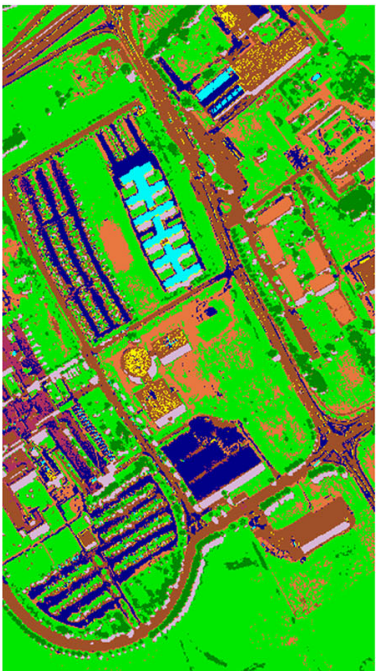

(f)

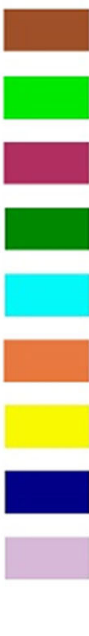

(d)

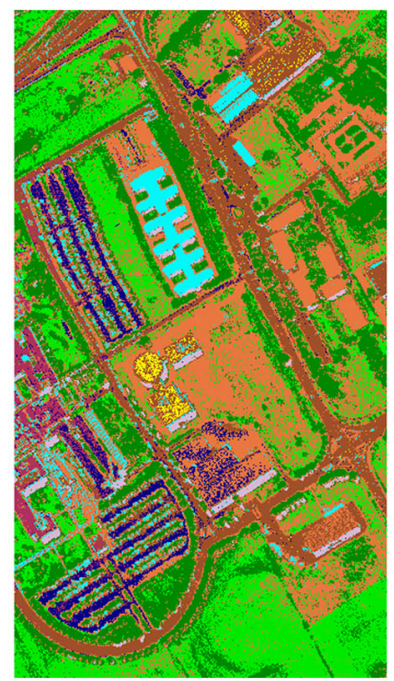

(g)

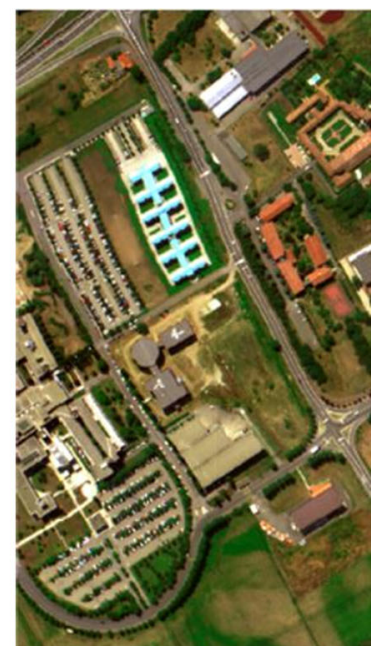

(b)

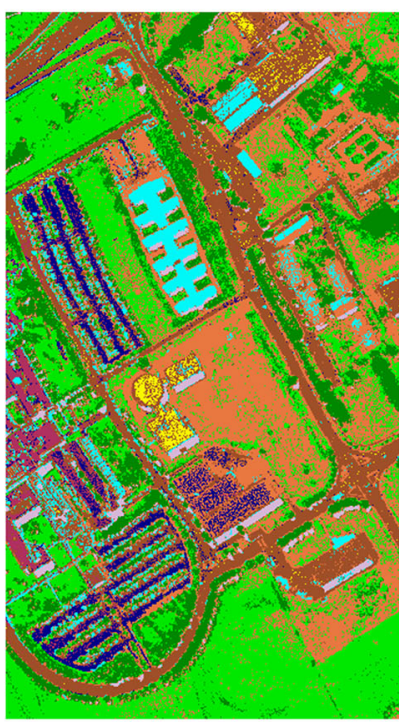

(e)

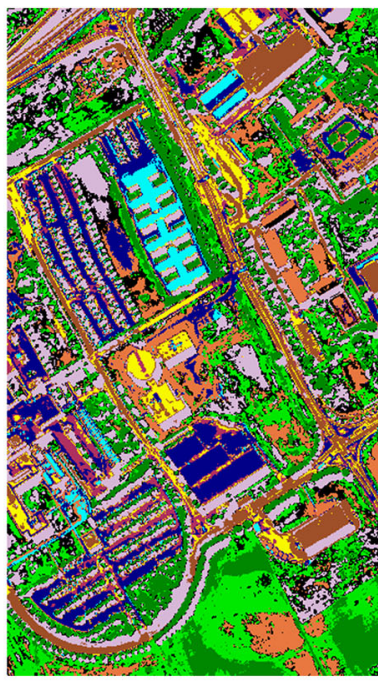

(h)

Figure 6. (a) The geographical location of Pavia University, (b) original image of Pavia University from ROSIS sensor, (c) reference data, (d) legend and classification map of (e) proposed method, (f) SVM, (g) traditional GFLVQ + proposed data reduction and (h) SAM. 
Note that to obtain a meaningful map, we used our proposed data reduction method (section 2.1.1) as a preprocessing step in the traditional GFLVQ method.

Tables 8 and 9 show the classification results (overall accuracy and total disagreement) compared to the proposed neuro-fuzzy system. These tables show the superiority of the proposed method in comparison with the kernel-based SVM, SAM and traditional GFLVQ.

As indicated in table 9, the total disagreement parameter for the proposed method, which is the sum of the quantity and allocation disagreements, is less than that of the other methods. This means that the spatial allocation of correct classes in the final map using the proposed method is the best.

In order to support the assertion that our new algorithm outperforms other methods, we consider the classification maps for Pavia University and Indian Pine obtained using our algorithm and using the traditional GFLVQ, SVM and SAM.

It can be seen that for the Pavia University dataset (figure 6), the classification map obtained by the proposed method (e) is more accurate than the others. For example, the classes of Trees and Meadows (which are mixed in the original image (b)) are better separated in the results for the proposed algorithm (e) than in those for SVM (f), traditional GFLVQ (g) and SAM (h) (see figure 7).

This is due to precise training of the classes' parameters, resulting in pixels being distinguished correctly. The main drawback of SAM, besides its poor accuracy, is that some pixels of the image are left unclassified (black parts in image (h)). SVM (f) and traditional GFLVQ (g) do not have this drawback, but they are not able to pick out some pixels very well, especially those which are spectrally similar.

In the case of the Indian Pine dataset, the final result of the proposed method is clearly more accurate than the other maps (as can be visually interpreted from figure 8). This is due to the special training phase of our neuro-fuzzy system which can distinguish between spectrally similar classes better than methods such as SVM, SAM and traditional GFLVQ. Note that the target classes of this dataset are not mutually exclusive, so we cannot expect to reach overall accuracies as high as in the first two datasets. Nevertheless, the neurofuzzy system outperforms the other methods.

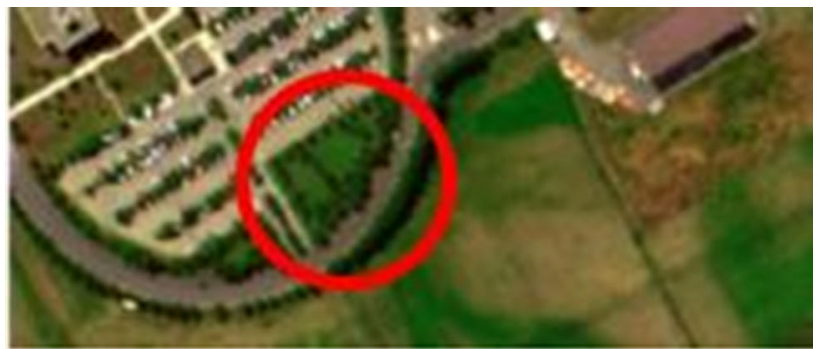

(a)

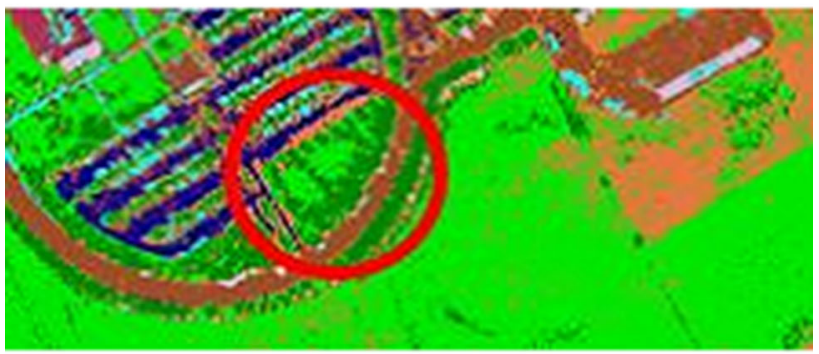

(b)

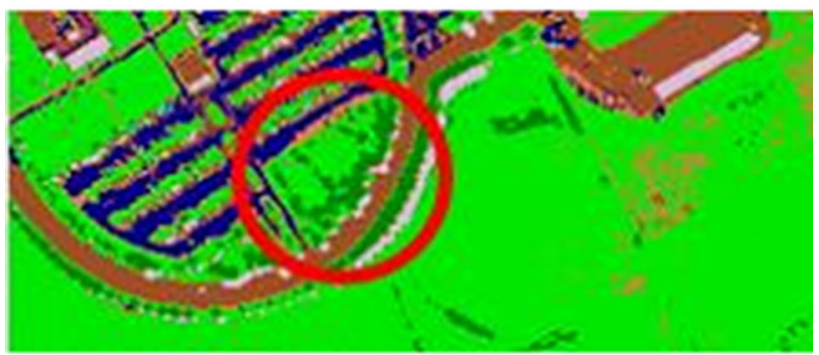

(c)

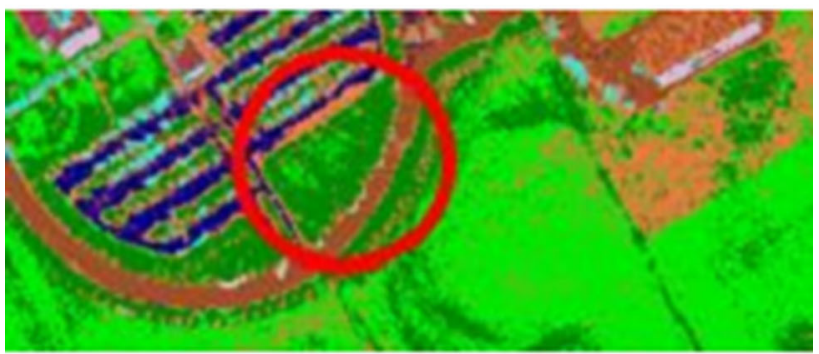

(d)

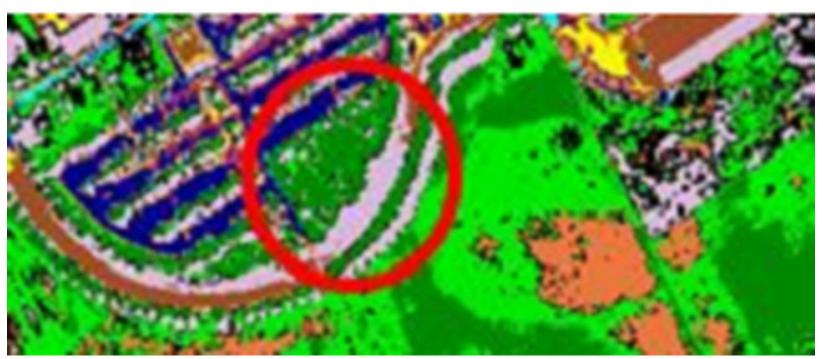

(e)

Figure 7. (a) A part of the original image which has two spectrally similar classes: meadows and trees. Classification map of (b) proposed method, (c) SVM, (d) traditional GFLVQ + proposed data reduction and (e) SAM for Pavia University dataset. 


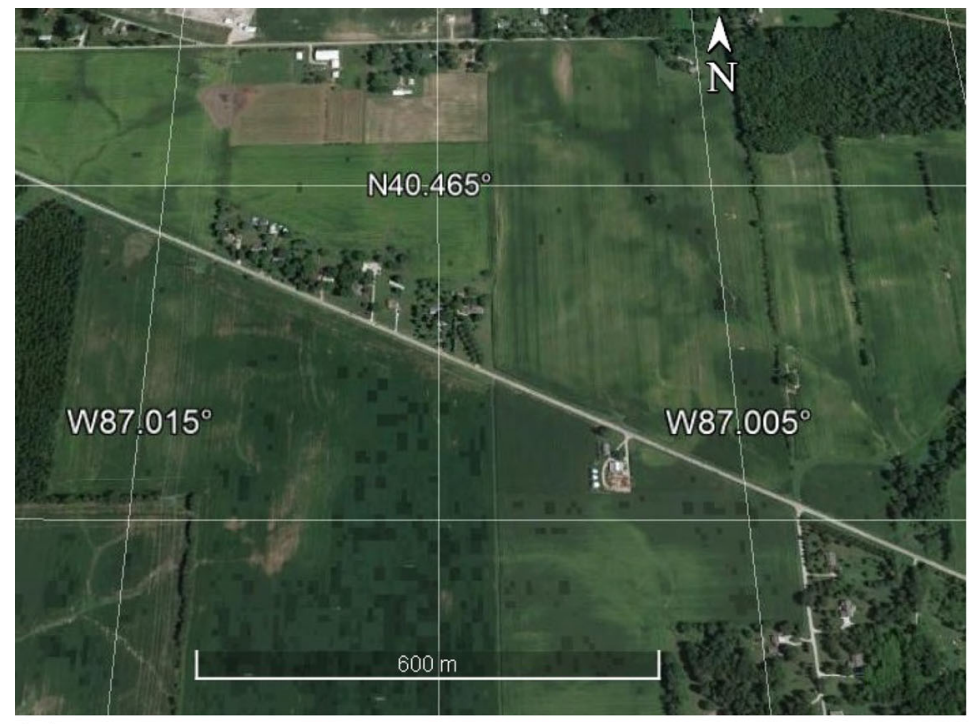

(a)

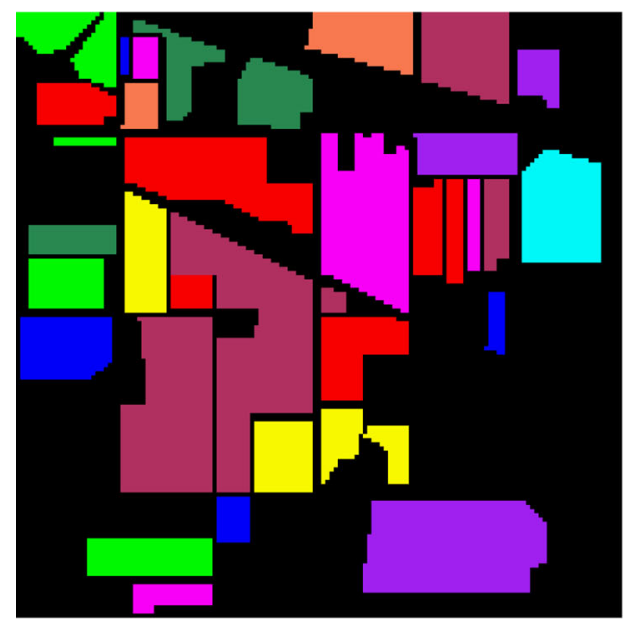

(c)

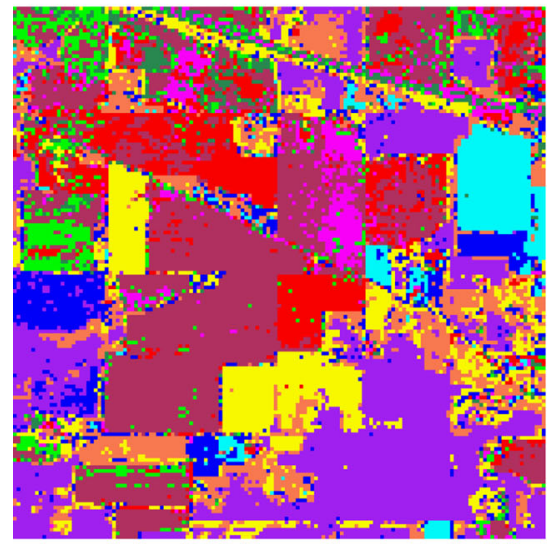

(f)

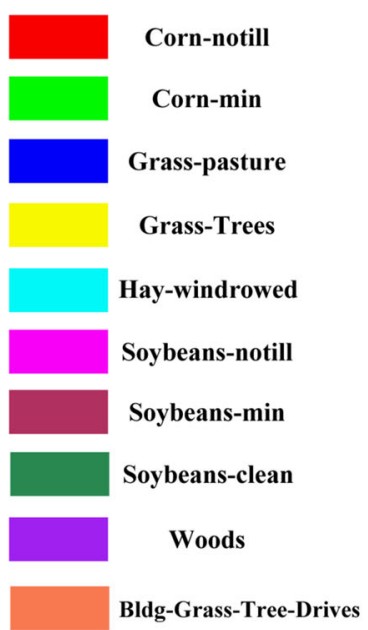

(d)

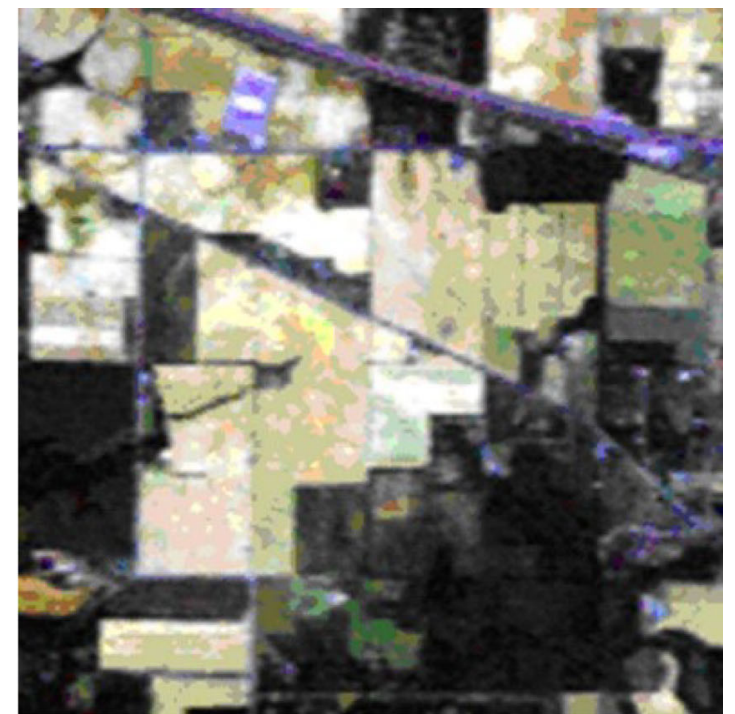

(b)

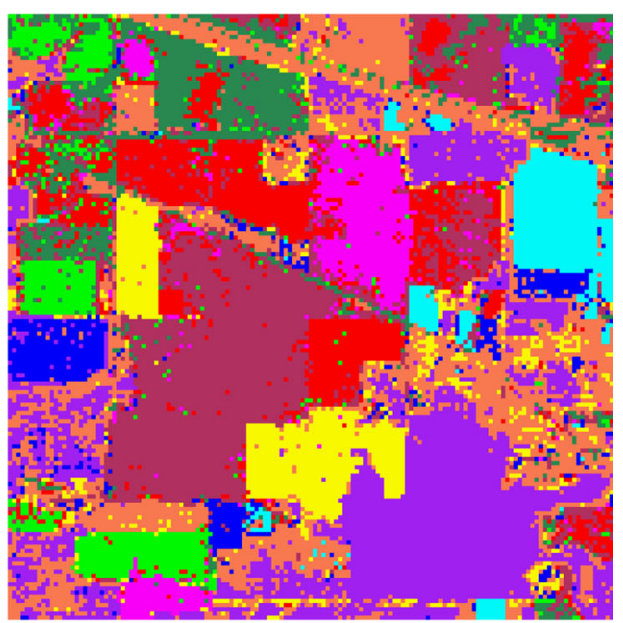

(e)

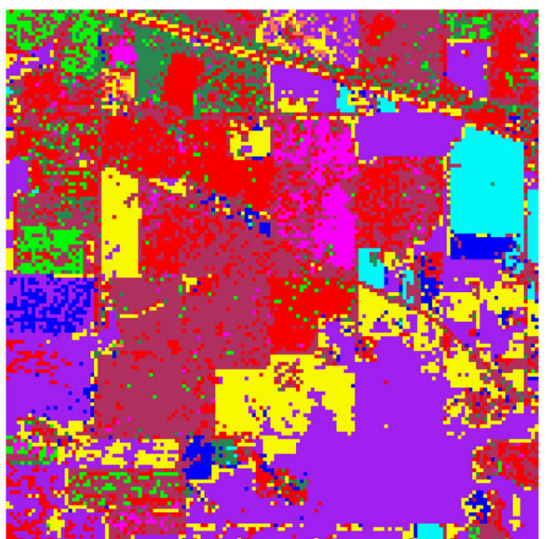

(g)

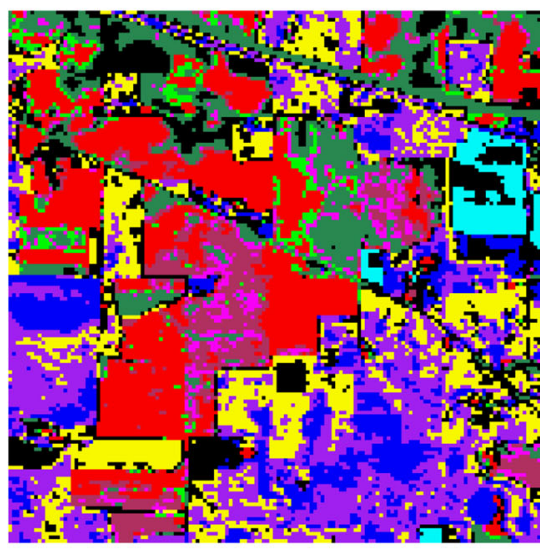

(h)

Figure 8. (a) The geographical location of Indian Pine, (b) original image of Indian Pine, (c) reference data, (d) legend and classification map of (e) proposed method, (f) SVM, (g) traditional GFLVQ + proposed data reduction and (h) SAM.

The proposed neuro-fuzzy system is powerful enough to separate adjacent classes. Figure 9 displays a small part of the reference image with two different classes (Corn - min and Soybean no till). Only the proposed method was able to successfully distinguish between these two classes. 


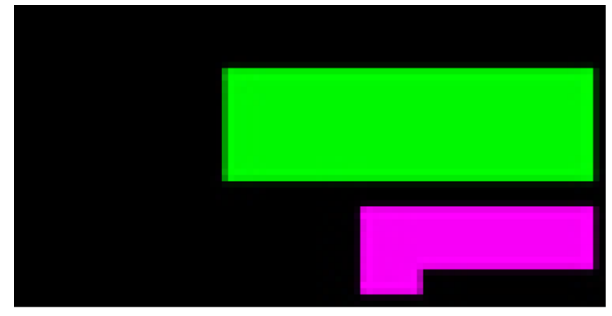

(a)

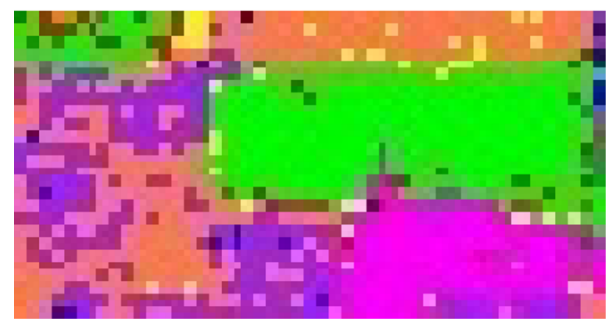

(b)

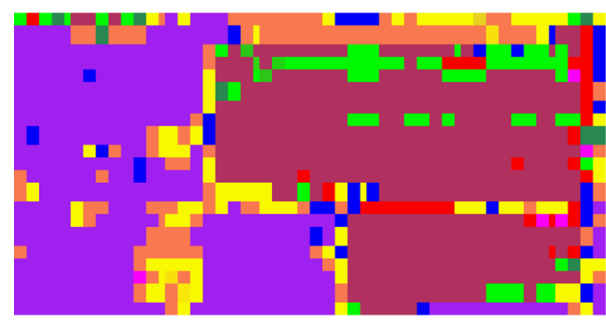

(c)

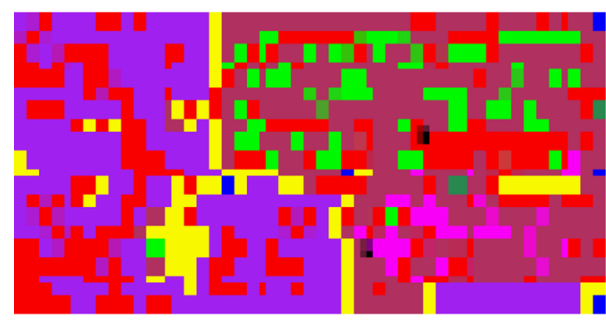

(d)

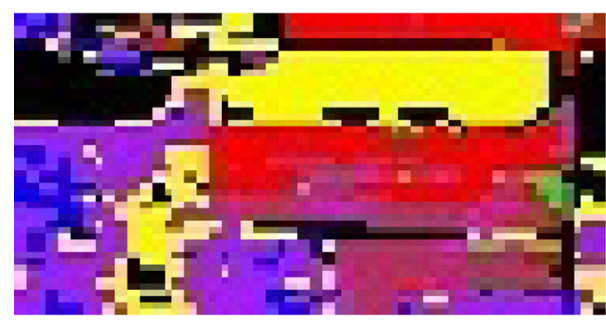

(e)

Figure 9. (a) A part of the original image which contains two different classes: corn - min (green) and soybeans - no till (magenta). Classification map of (b) proposed method, (c) SVM, (d) traditional GFLVQ + proposed data reduction and (e) SAM for Indian Pine dataset.

As the Botswana dataset is basically a rural image, most of the classes are vegetation which may be unfamiliar. Therefore, we decided to use only the classification maps of Pavia University and Indian Pine, since the objects and classes of these image can be visually understood, and further analysis of the maps can be explained.

\section{Conclusion}

Hyperspectral data classification procedures face several problems, including an insufficient number of training samples compared with the huge number of bands and the curse of dimensionality. Therefore, traditional classification methods are incapable of producing accurate results. Intelligent systems are one of the main alternative methods that can be used in place of traditional classification methods. Among intelligent systems, neural networks and fuzzy systems have many advantages and have the potential to be used in remote sensing applications.

In this research, these two methods were applied to overcome the shortcomings of traditional classifiers. Thus, a new neuro-fuzzy system has been proposed. The system can design fuzzy if-then rules to classify hyperspectral images correctly. The required parameters of these rules are obtained from training via the learning algorithm of the neural network. After the learning phase, the trained parameters could be applied to classify any kind of data. The proposed system takes advantage of LVQ fuzzy networks with only one neuron for each class, giving it the benefits of both supervised and unsupervised training. This system produces excellent results by adding normalisation and data reduction steps in the PCA-based preprocessing. The preprocessing procedure compresses data based on information on classes.

The proposed system was applied to the classification of three sets of data: Pavia University from ROSIS, Botswana from Hyperion and Indian Pine from AVIRIS. The number of PCA bands and the amount of training data were specified individually for each dataset to allow a fair comparison. The final thematic maps were compared with the results of SAM and SVM classification methods which are commonly used in the remote sensing community. The comparison was performed using two different types of accuracy assessment measures, $\kappa$ coefficients and disagreements. Quantitative comparison of the metrics proved that the new system outperforms its counterparts. A more than 5\% increase in overall accuracy for the Pavia University image was observed. The corresponding figures for the Botswana and Indian Pine images were $2 \%$ and $7 \%$, respectively. 
It can, therefore, be said that the proposed neuro-fuzzy system can overcome the shortcomings of traditional classification methods for hyperspectral images. In order to obtain precise results from this system, the number and distribution of the training samples should be sufficient for the purpose, especially in the case of complicated images.

\section{References}

Aghaee R and Mokhtarzade M 2015 Classification of hyperspectral images using subspace projection feature space; IEEE Geosci. Remote Lett. 12(9) 1803-1807.

Alajlan N, Bazi Y, Melgani F and Yager R R 2012 Fusion of supervised and unsupervised learning for improved classification of hyperspectral images; Inform. Sci. 217 39-55.

Arslan O 2009 A novel confidence estimation method for neural networks in multispectral image classification; Int. J. Dig. Earth 2(4) 343-358.

Benítez J M, Castro J L and Requena I 1997 Are artificial neural networks black boxes?; IEEE Trans. Neural Netw. 8(5) 1156-1164.

Bilgin G, Erturk S and Yildirim T 2008 Unsupervised classification of hyperspectral-image data using fuzzy approaches that spatially exploit membership relations; IEEE Geosci. Remote Lett. 5(4) 673-677.

Bishop C M 2006 Pattern recognition and machine learning; Springer, New York.

Borasca B, Bruzzone L, Carlin L and Zusi M 2006 A fuzzyinput fuzzy-output SVM technique for classification of hyperspectral remote sensing images; In: Proceedings of the 7th Nordic Signal processing symposium, NORSIG 2006, pp. 2-5.

Braspenning P J and Thuijsman F 1995 Artificial neural network, an introduction to ANN theory and practice; Springer, Berlin.

Chi H-M and Ersoy O K 2005 A statistical self-organizing learning system for remote sensing classification; IEEE Trans. Geosci. Remote 43(8) 1890-1900.

Dennison P E and Roberts D A 2003 Endmember selection for multiple endmember spectral mixture analysis using endmember average RMSE; Remote Sens. Environ. 87(2) $123-135$

Dennison P E, Halligan K Q and Roberts D A 2004 A comparison of error metrics and constraints for multiple endmember spectral mixture analysis and spectral angle mapper; Remote Sens. Environ. 93(3) 359-367.

Franke J, Roberts D A, Halligan K and Menz G 2009 Hierarchical multiple endmember spectral mixture analysis (MESMA) of hyperspectral imagery for urban environments; Remote Sens. Environ. 113(8) 1712-1723.

Hughes G 1968 On the mean accuracy of statistical pattern recognizers; IEEE Trans. Inform. Theory 14(1) 55-63.

Jimenez L O, Morales-Morell A and Creus A 1999 Classification of hyperdimensional data based on feature and decision fusion approaches using projection pursuit, majority voting, and neural networks; IEEE Geosci. Remote Lett. 37(3) 1360-1366.
Jolliffe I 2002 Principal component analysis; John Wiley and Sons, Ltd.

Kohonen T 1990 The self-organizing map; Proc. IEEE 78(9) 1464-1480.

Kumar T, Prasad R, Choudhary A, Mishra V N, Gupta D K and Srivastava P K 2016 A statistical significance of differences in classification accuracy of crop types using different classification algorithms; Geocarto Int. 32(2) 206-224.

Kuo B-C, Huang W-C, Liu H-C and Tseng S-C 2008 A novel fuzzy c-means method for hyperspectral image classification; In: Geoscience and remote sensing symposium 2008, IGARSS 2008, IEEE International 2, II-1002.

Mehrotro K, Mohan C K and Ranka S 2000 Elements of artificial neural networks (2nd edn), MIT Press, Cambridge.

Mojaradi B, Abrishami-Moghaddam H, Zoej M J V and Duin R P 2009 Dimensionality reduction of hyperspectral data via spectral feature extraction; IEEE Trans. Geosci. Remote 47(7) 2091-2105.

Nauck D, Klawonn F and Kruse R 1997 Foundations of neuro-fuzzy systems; John Wiley \& Sons, Ltd.

Neuenschwander A L, Crawford M M and Ringrose S 2005 Results from the EO-1 experiment - A comparative study of earth observing-1 advanced land imager (ALI) and Landsat ETM + data for land cover mapping in the Okavango delta, Botswana; Int. J. Remote 26(19) 4321-4337.

Nomura T and Miyoshi T 1995 An adaptive rule extraction with the fuzzy self-organizing map and a comparison with other methods; In: Third international symposium on uncertainty modeling and analysis, 1995, and annual conference of the North American Fuzzy Information Processing Society, Proceedings of ISUMA-NAFIPS'95.

Petropoulos G P, Kalivas D P, Georgopoulou I A and Srivastava P K 2015 Urban vegetation cover extraction from hyperspectral imagery and geographic information system spatial analysis techniques: Case of Athens, Greece J. Appl. Remote Sens. 9096088.

Picón A, Ghita O, Whelan P F and Iriondo P M 2009 Fuzzy spectral and spatial feature integration for classification of nonferrous materials in hyperspectral data; IEEE Trans. Ind. Inform. 5(4) 483-494.

Pontius Jr R G and Millones M 2011 Death to Kappa: Birth of quantity disagreement and allocation disagreement for accuracy assessment; Int. J. Remote 32(15) 4407-4429.

Qiu F 2008 Neuro-fuzzy based analysis of hyperspectral imagery; Photogramm. Eng. Remote Sens. 74(10) 12351247.

Qiu F and Jensen J 2004 Opening the black box of neural networks for remote sensing image classification; Int. J. Remote 25(9) 1749-1768.

Ratle F, Camps-Valls G and Weston J 2010 Semisupervised neural networks for efficient hyperspectral image classification; IEEE Trans. Geosci. Remote 48(5) 2271-2282.

Richards J A 2013 Remote sensing digital image analysis (5th edn), Springer, Canberra.

Singh S K, Srivastava P K, Gupta M, Thakur J K and Mukherjee S 2014 Appraisal of land use/land cover of mangrove forest ecosystem using support vector machine; Environ. Earth Sci. 71 2245-2255.

Somers B, Asner G P, Tits L and Coppin P 2011 Endmember variability in spectral mixture analysis: A review; Remote Sens. Environ. 115(7) 1603-1616. 
Song C 2005 Spectral mixture analysis for subpixel vegetation fractions in the urban environment: How to incorporate endmember variability?; Remote Sens. Environ. 95(2) 248-263.

Srivastava P K, Han D, Rico-Ramirez M A, Bray M and Islam T 2012 Selection of classification techniques for land use/land cover change investigation; Adv. Space Res. 50 1250-1265.

Su M and Tew C 2000 A self-organizing feature-map-based fuzzy system; In: Proceedings of the IEEE-INNS-ENNS international joint conference on neural networks, IJCNN 2000, pp. 20-25.

Torabi A J, Er M J, Lim B S, Zhai L, Oentaryo R J, Peen G O and Zurada J M 2015 A surveyon arti- ficial intelligence-based modeling techniques for high speed milling processes; IEEE Syst. J. 9(3) 10691080.

Yamany S M, Farag A A and Hsu S-Y 1999 A fuzzy hyperspectral classifier for automatic target recognition (ATR) systems; Pattern Recogn. Lett. 20(11) 1431-1438.

Zhang J and Foody G 2001 Fully-fuzzy supervised classification of sub-urban land cover from remotely sensed imagery: Statistical and artificial neural network approaches; Int. J. Remote 22(4) 615-628.

Zhang C and Xie Z 2012 Combining object-based texture measures with a neural network for vegetation mapping in the Everglades from hyperspectral imagery; Remote Sens. Environ. 124 310-320.

Corresponding editor: Prasanth K SRIVAstava 